University of Nebraska - Lincoln

DigitalCommons@University of Nebraska - Lincoln

$5-2-2008$

\title{
Wavelength Scaling of High-Harmonic Yield: Threshold Phenomena and Bound State Symmetry Dependence
}

\author{
M. V. Frolov \\ Voronezh State University, Voronezh 394006, Russia \\ N. L. Manakov \\ Voronezh State University, manakov@phys.vsu.ru \\ Anthony F. Starace \\ University of Nebraska-Lincoln, astarace1@unl.edu
}

Follow this and additional works at: https://digitalcommons.unl.edu/physicsstarace

Part of the Physics Commons

Frolov, M. V.; Manakov, N. L.; and Starace, Anthony F., "Wavelength Scaling of High-Harmonic Yield: Threshold Phenomena and Bound State Symmetry Dependence" (2008). Anthony F. Starace Publications. 131.

https://digitalcommons.unl.edu/physicsstarace/131

This Article is brought to you for free and open access by the Research Papers in Physics and Astronomy at DigitalCommons@University of Nebraska - Lincoln. It has been accepted for inclusion in Anthony F. Starace Publications by an authorized administrator of DigitalCommons@University of Nebraska - Lincoln. 


\title{
Wavelength Scaling of High-Harmonic Yield: Threshold Phenomena and Bound State Symmetry Dependence
}

\author{
M. V. Frolov, ${ }^{1}$ N. L. Manakov, ${ }^{1}$ and Anthony F. Starace ${ }^{2}$ \\ ${ }^{1}$ Department of Physics, Voronezh State University, Voronezh 394006, Russia \\ ${ }^{2}$ Department of Physics and Astronomy, The University of Nebraska, Lincoln, Nebraska 68588-0111, USA
}

(Received 29 November 2007; published 1 May 2008)

\begin{abstract}
Describing harmonic generation (HG) in terms of a system's complex quasienergy, the harmonic power $P_{\Delta E}(\lambda)$ (over a fixed interval, $\Delta E$, of harmonic energies) is shown to reproduce the wavelength scaling predicted recently by two groups of authors based on solutions of the time-dependent Schrödinger equation: $P_{\Delta E}(\lambda) \sim \lambda^{-x}$, where $x \approx 5-6$. Oscillations of $P_{\Delta E}(\lambda)$ on a fine $\lambda$ scale are then shown to have a quantum origin, involving threshold phenomena within a system of interacting ionization and HG channels, and to be sensitive to the bound state wave function's symmetry.
\end{abstract}

PACS numbers: $32.80 . \mathrm{Rm}, 42.65 . \mathrm{Ky}$

Owing to its important applications, high-harmonic generation (HHG) is a main focus of intense laser-atom physics. The existence of a plateau in the spectrum of harmonic rates allows the generation of intense coherent radiation in the extreme vacuum ultraviolet (vuv) and soft x-ray region, thus opening the possibility of exploring nonlinear optical processes in this region [1]. The HHG process is also a fundamental component of attosecond science [2], allowing the generation of attosecond pulses. The well-known $\left|E_{0}\right|+3.17 u_{p}$ law for the energy position of the HHG plateau cutoff (where $\left|E_{0}\right|$ is the atomic binding energy, $u_{p}=e^{2} F^{2} /\left(4 m \omega^{2}\right)$ is the ponderomotive energy, and $F$ and $\omega$ are the laser field amplitude and frequency) shows that use of midinfrared lasers (e.g., of wavelength $\sim 2 \mu \mathrm{m}$ [3]) allows the generation of higher harmonics. The key issue is the harmonic yield for such wavelengths as compared to that for the commonly-used Ti:sapphire laser, with $\lambda \approx 0.8 \mu \mathrm{m}$, i.e., the wavelength scaling law for the HHG efficiency. According to the semiclassical strong-field approximation (SFA) [4], this scaling law should be $\sim \lambda^{-3}$, for which there is partial experimental support [5]. However, a much faster decrease of the HHG efficiency between 0.8 and $2.0 \mu \mathrm{m}\left(\sim \lambda^{-x}\right.$, where $\left.x \approx 5-6\right)$ has been predicted recently by two groups of authors $[3,6]$ based on numerical solutions of the time-dependent Schrödinger equation (TDSE). Moreover, Ref. [6] predicts that the wavelength dependence is not smooth on a fine $\lambda$ scale, but exhibits rapid oscillations, which are interpreted in terms of interference of up to five returning electron trajectories. References $[3,6]$ both note that the usual semiclassical SFA treatment of HHG in terms of two classical trajectories ("short" and "long") fails to explain these phenomena: Ref. [3] notes the "unexpectedly important role" of higher order classical trajectories; Ref. [6] remarks that "the frequently discussed short and long trajectories...are insufficient to account for the interference oscillations."

In this Letter we examine the issue of $\lambda$-scaling of the HHG yield for a short-range potential model system that has the virtues that (i) completely quantum predictions for the HHG yield can be obtained essentially exactly and (ii) results can be obtained relatively easily over a wide range of the system parameters, since much of the theory is analytical. The results of analysis of the $\lambda$-scaling of the HHG yield for our model system agree both qualitatively and, where possible, quantitatively, with the TDSE results in Refs. [3,6], thereby providing evidence of the generality of these strong-field phenomena. Moreover, our approach provides a fundamental quantum explanation for the rapid oscillations found numerically (and interpreted semiclassically) in Ref. [6]. In our approach, these oscillations are due to effects of threshold phenomena (TP) $[7,8]$ within a system of interacting multiphoton ionization and harmonic generation channels. In view of this explanation, the necessity for including many interfering classical trajectories to reproduce the TDSE results [6] is not surprising, since the standard semiclassical approach cannot describe purely quantum effects, such as, in particular, those related to TP.

We employ our recently developed ab initio quantum formulation for the HHG amplitude in terms of the complex quasienergy of a bound electron in a monochromatic field [9]; i.e., it does not require computation of the system's wave function. Combined with the time-dependent effective range (TDER) theory [10], this formulation allows one to obtain exact results for HHG rates for an electron bound by short-range forces. Moreover, our results for this model system may be scaled to describe the HHG yield for neutral atoms, in particular, for $\mathrm{Ar}$ and $\mathrm{H}$, in order to compare our results directly with those obtained in Refs. $[3,6]$ at fixed intensity, $1.6 \times 10^{14} \mathrm{~W} / \mathrm{cm}^{2}$.

Let $\epsilon_{|m|}(F, \omega)$ be the complex quasienergy of a ground state atom with energy $E_{0}$, angular momentum $l$, and projection $m$, in a linearly polarized electric field $\mathbf{F}(t)=$ $\hat{\mathbf{z}} F \cos \omega t$. As shown in Ref. [9], the amplitude $\left(\chi_{n}^{(|m|)}\right)$ and rate $\left(\mathcal{R}_{n}\right)$ for generating the $n$th harmonic are

$$
\chi_{n}^{(|m|)}(F, \omega)=-\left.2 \frac{\partial \Delta \epsilon_{|m|}}{\partial F_{h}}\right|_{F_{h}=0},
$$




$$
\mathcal{R}_{n}=\frac{(n \omega)^{3}}{8 \pi \hbar c^{3}} \frac{1}{2 l+1} \sum_{m}\left|\chi_{n}^{(|m|)}(F, \omega)\right|^{2},
$$

where $\Delta \epsilon_{|m|}$ is the lowest-order (linear in $F_{h}$ ) correction to $\epsilon_{|m|}(F, \omega)$ in a weak (probe) field, $\mathbf{F}_{h}(t)=\hat{\mathbf{z}} F_{h} \cos \Omega t$, having the harmonic frequency, $\Omega=n \omega$. The results in Eqs. (1) and (2), are general, ab initio quantum definitions of the single-atom HHG amplitude and rate [9]; however, numerical calculations of $\Delta \epsilon_{|m|}$ for real atoms are difficult. Within the single active electron approximation, we employ here the TDER theory [10] for HHG calculations employing Eqs. (1) and (2), as described in Ref. [11].

As in Refs. [3,6], we present $\lambda$-scaling results for the wavelength interval 0.8-2.0 $\mu \mathrm{m}$. As shown in Ref. [11], for this wavelength range the exact TDER results for $\chi_{n}^{(|m|)}(F, \omega)$ are in perfect agreement with those in the "Keldysh approximation (KA)" for HHG. This approximation is very similar to the KA (or SFA) result for ionization (see Refs. [11,12] for details) and depends on only two atomic parameters, the binding energy $\left|E_{0}\right|$ and the angular momentum $l$ of the initially bound electron. We apply the TDER theory to model $\mathrm{HHG}$ rates for $\mathrm{H}$ and $\mathrm{He}$ (with active electrons in $s$-states) and $\mathrm{Ar}$ (with the active electron in a $p$-state). Concerning the parameter $\left|E_{0}\right|$, we note that the excited states of a bound electron are neglected in the TDER model, while the high-lying atomic states are strongly distorted by an intense low-frequency field and form a quasicontinuum of broadened Floquet states, effectively lowering the ionization potential, $I_{a}$ : $\tilde{I}_{a}=I_{a}-\Delta_{a}$. For $I=1.6 \times 10^{14} \mathrm{~W} / \mathrm{cm}^{2}$, our estimates of tunneling ionization rates for hydrogen show that beginning from levels with $n=3$ the ionization width exceeds the distance between neighboring levels. Thus in our calculations we choose the effective ionization potential $\tilde{I}_{H}=$ $10.5 \mathrm{eV}$ for hydrogen [cf. the discussion of Eq. (8) below]. For consistency, we use approximately the same percentage reductions of $I_{a}$ for $\mathrm{He}$ and $\operatorname{Ar}: \tilde{I}_{\mathrm{He}}=19.0 \mathrm{eV}$ and $\tilde{I}_{\mathrm{Ar}}=13.0 \mathrm{eV}$.

We define the integrated HHG efficiency as the harmonic power radiated in a fixed energy interval of the HHG spectrum, $\Delta E=\left(n_{f}-n_{i}\right) \hbar \omega$,

$$
P_{\Delta E}(\lambda)=\hbar \omega \sum_{n_{i}}^{n_{f}} n \mathcal{R}_{n},
$$

for which the TDSE studies in the interval $\Delta E=$ $20-50 \mathrm{eV}$ yield the following wavelength scaling law $[3,6]$,

$$
P_{\Delta E}(\lambda) \sim \lambda^{-x}, \quad x \approx 5-6 .
$$

Our results for $x$ are given in Table I for three intensities (in units of $10^{14} \mathrm{~W} / \mathrm{cm}^{2}$ ), $I=1.6,3.2$, and 4.45 , and several values of $\Delta E$. The results for $x$ are similar for different $\Delta E$ and decrease slowly when $I$ increases. For $I=1.6$, our result for Ar coincides with that of Ref. [3], while the results of [6] for $\mathrm{H}$ and $\mathrm{Ar}$ are about $10 \%$ less than ours.
TABLE I. Values of $x$ [cf. Eq. (4)] vs laser intensity (in units of $\left.10^{14} \mathrm{~W} / \mathrm{cm}^{2}\right)$ and energy interval $\Delta E$.

\begin{tabular}{lccccc}
\hline \hline I & $\Delta E(\mathrm{eV})$ & Ref. & $\mathrm{H}$ & $\mathrm{He}$ & $\mathrm{Ar}$ \\
\hline 1.6 & $20-50$ & Present & 5.3 & 5.5 & 6.0 \\
& $20-50$ & {$[3]$} & $\ldots$ & $\cdots$ & 6.0 \\
& $20-50$ & {$[6]$} & 4.8 & $\ldots$ & 5.5 \\
3.2 & $20-50$ & Present & 5.0 & 5.2 & 5.4 \\
& $20-70$ & Present & 5.0 & 5.3 & 5.3 \\
& $20-90$ & Present & 4.9 & 5.4 & 5.2 \\
4.45 & $40-80$ & Present & 4.7 & 5.0 & 5.0 \\
& $40-80$ & {$[3]$} & $\cdots$ & 5.0 & $\cdots$ \\
\hline \hline
\end{tabular}

The $\lambda$-dependence of $P_{\Delta E}$ for $\mathrm{H}$ and Ar in Fig. 1(a) calculated with a $\lambda$-spacing of $50 \mathrm{~nm}$ is similar to that in Ref. [6]. The generality of Eq. (4) is supported by the fact, that our values of $x$ in Table I are insensitive to the choice of $\tilde{I}_{a}$ and remain the same using unshifted ionization potentials $I_{a}$ for $\mathrm{H}, \mathrm{He}$, and $\mathrm{Ar}$ [in contrast to the behavior of $P_{\Delta E}(\lambda)$ on a fine $\lambda$ scale; see below]. Also, the agreement between TDER and TDSE results indicates that the excited atomic states (omitted in the TDER theory) do not affect the scaling law (4).

Our analysis provides an explanation for the much faster (than $\sim \lambda^{-3}$ ) decrease of $P_{\Delta E}(\lambda)$ with increasing $\lambda$. According to saddle-point estimates of our analytical result for the HHG amplitude for an $s$-state (in terms of integrals of Bessel functions [11]), the $\lambda^{-3}$ law originates from the contribution of the least-spreading, shortest classical trajectory and is applicable only for harmonics near the plateau cutoff (see also Refs. [13,14]). However, the energy interval $\Delta E$ considered in Ref. [3] (as well as in Table I) lies below the cutoff region. Since the standard (two-term) SFA fails to describe the onset and middle parts of the HHG plateau, the faster than $\lambda^{-3}$ decrease of $P_{\Delta E}(\lambda)$ found in Ref. [3] is not surprising. Indeed, in terms of classical trajectories, those with longer return times (and larger spreadings) contribute below the cutoff region, thus leading to a value of $x$ larger than 3. In fact, our representative TDER calculations of the HHG yields for fixed numbers of

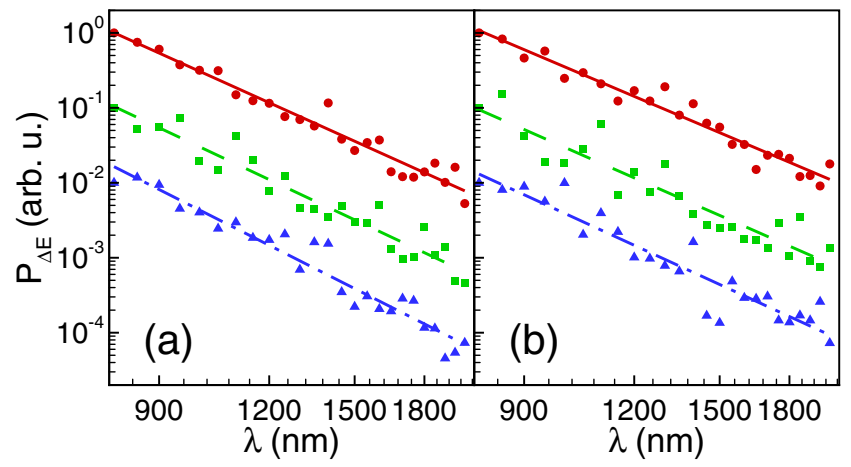

FIG. 1 (color online). Wavelength dependence of $P_{\Delta E}(\lambda)$ for $\mathrm{H}$ (circles), He (squares), and Ar (triangles) for $\Delta E=20-50 \mathrm{eV}$ and $I=$ (a) $1.6 \times 10^{14} \mathrm{~W} / \mathrm{cm}^{2}$ or (b) $3.2 \times 10^{14} \mathrm{~W} / \mathrm{cm}^{2}$. Lines are fits of the scaling law (4) to the data, with $x$ given in Table I. 


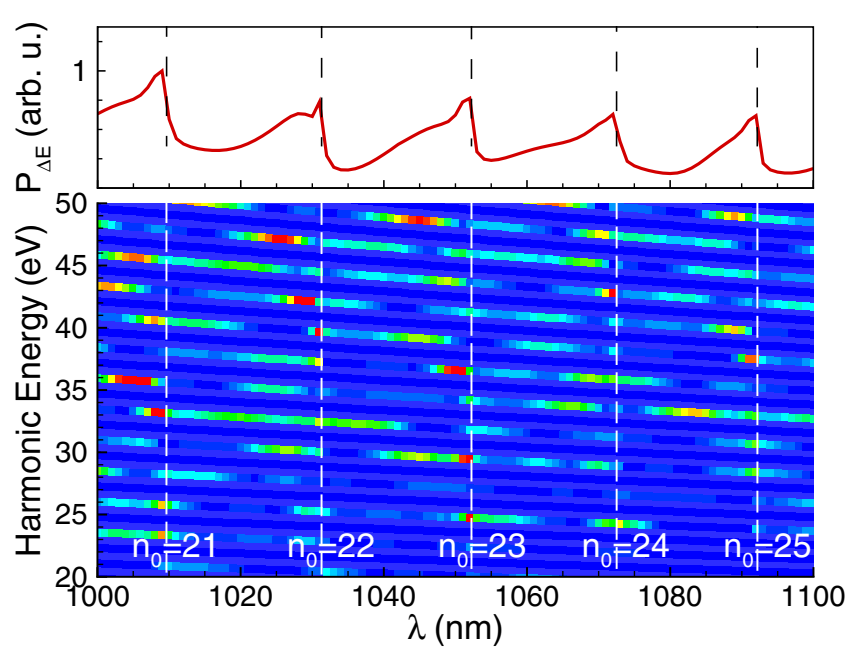

FIG. 2 (color online). Threshold phenomena in $P_{\Delta E}(\lambda)$ (upper panel) and in the yields of the individual harmonics in the interval $20-50 \mathrm{eV}$ (bottom panel) for the $\mathrm{H}$ atom, with $I=$ $1.6 \times 10^{14} \mathrm{~W} / \mathrm{cm}^{2}$. (cf. Fig. 2 in Ref. [6]).

harmonics within an interval $\Delta E$ have a scaling $\sim \lambda^{-(6-9)}$. Nevertheless, the results (cf. Table I) for the efficiency integrated over a fixed energy interval confirm the scaling law (4), which itself is only an approximate (large $\lambda$ scale) version of the more intriguing behavior of $P_{\Delta E}(\lambda)$ on a fine $\lambda$ scale.

The results for $P_{\Delta E}(\lambda)$ for $\mathrm{H}$ and $\mathrm{Ar}$ on a mesh with $\Delta \lambda=1 \mathrm{~nm}$ are given in Figs. 2 and 3. They exhibit oscillations similar to those revealed in Ref. [6]. Our analysis shows that the maxima of these oscillations correspond to thresholds of the lowest open ( $n_{0}$-photon) ionization channels, which are defined by the condition

$$
\operatorname{Re} \epsilon-u_{p}+n_{0} \hbar \omega=0,
$$

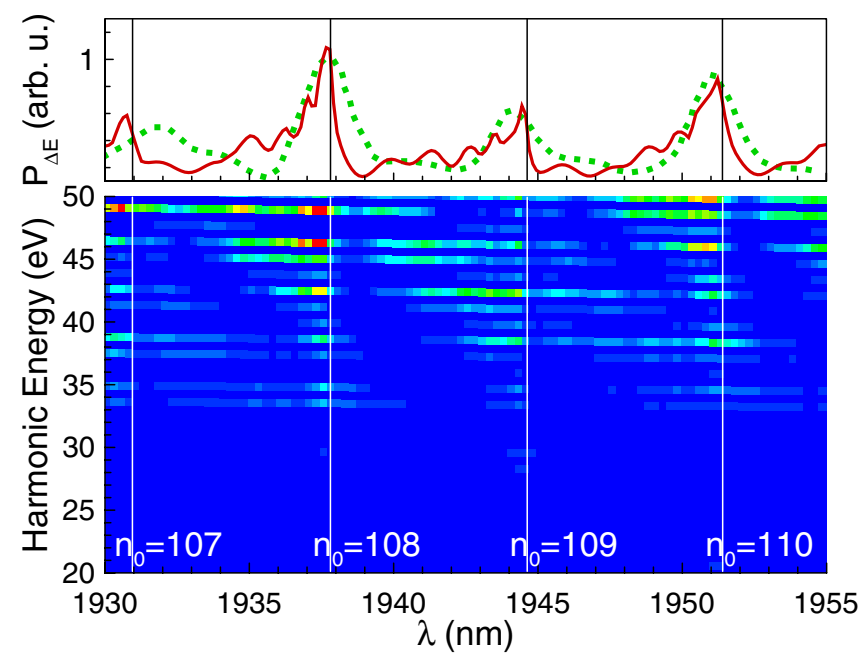

FIG. 3 (color online). The same as in Fig. 2, but for Ar over a different range of $\lambda$. In the upper panel, our results (solid line) are compared with the TDSE results [6] (dotted line). where $n_{0}$ is a positive integer and $\operatorname{Re} \epsilon \approx E_{0}$. According to the general theory of TP $[7,8]$ (see also Refs. [15,16]), TP in HHG consist in modifications of the wavelength dependences of the HHG rates, $\mathcal{R}_{n}$, at the closing or opening of an ATI channel $\left(n_{0}\right)$. These modifications result from nonanalyticities of the HHG amplitudes $\chi_{n}^{(|m|)}(F, \omega)$ at the corresponding threshold wavelength, $\lambda=\lambda_{n_{0}}(I)$.

As shown in Refs. [15,16], the TP in the rates $\mathcal{R}_{n}$ for different $n$ at a fixed wavelength $\lambda_{n_{0}}(I)$ are most pronounced at even (odd) $n_{0}$ for the case of an $s(p)$ initial bound state of the active electron (since the amplitudes $\chi_{n}^{(|m|)}(F, \omega)$ in this case have square-root branch point singularities, $\sim \sqrt{E-E_{\mathrm{th}}}$, that result in known "cusp" or "steplike" threshold anomalies [7,8]). What is surprising in Figs. 2 and 3 is that enhancements in the integrated yield, $P_{\Delta E}(\lambda)$, appear for both even and odd $n_{0}$. The explanation of this result is that for odd (even) $n_{0}$ for the case of $s(p)$ initial states, the amplitudes $\chi_{n}^{(|m|)}(F, \omega)$ have high-order branch point singularities $\left[\sim\left(E-E_{\mathrm{th}}\right)^{k+1 / 2}\right.$, $k \geq 1$ ], which lead to much less pronounced modifications of $\mathcal{R}_{n}$ at $\lambda=\lambda_{n_{0}}(I)$, but which nevertheless exist (and are visible [15]). In summing over $n$ to obtain the integrated yield, $P_{\Delta E}(\lambda)$, these small modifications of $\mathcal{R}_{n}$ for a given $n$ demonstrate a remarkable "coherence", resulting in enhancements of $P_{\Delta E}$ comparable to those for the square-root singularities. These TP thus reveal the fundamental origin of the oscillations in $P_{\Delta E}(\lambda)$, which were discussed in Ref. [6] in terms of interferences between electron trajectories. Moreover, it is somewhat surprising that our simple model is able to describe not only the general shapes of $P_{\Delta E}(\lambda)$ for both $\mathrm{H}$ and $\mathrm{Ar}$ on a fine $\lambda$ scale, but also reproduces such details as the alternations in the heights of the peaks for Ar (cf. Fig. 3). This difference from the flatter peak heights in $\mathrm{H}$ (cf. Fig. 2) results from differences in the shapes of HHG spectra as functions of harmonic number $n$ for different ( $s$ or $p$ ) spatial symmetries of the respective ground state wave functions [11]. (This difference is caused, in part, by the relative increase of the intensities of harmonics near the cutoff with increasing $\lambda$ in the case of $p$-states [11]; this increase is seen also in Fig. 1(b) of Ref. [3].) Well-resolved substructures in the $\lambda$-dependence of $P_{\Delta E}(\lambda)$ in Fig. 3 are due to the fact that the rates $\mathcal{R}_{n}$ for $p$-states are relatively small in the first third of the plateau [11] (cf. the bottom panel in Fig. 3); thus the dominant contributions to $P_{\Delta E}(\lambda)$ come from only a limited set of harmonics near the upper end of the $\Delta E$ interval. (Apparently, the Fourier broadening of the lowfrequency few-cycle laser pulse smears these substructures in the TDSE results [6]).

The intervals, $\delta \lambda$, between peak values of $P_{\Delta E}(\lambda)$ in Figs. 2 and 3 are the intervals between two neighboring "threshold wavelengths" [cf. Eq. (5)], $\lambda_{n_{i}}(I)$ and $\lambda_{n_{i}+1}(I)$, corresponding to the closing of the $n_{i}$ th and $\left(n_{i}+1\right)$ th ionization channels:

$$
\delta \lambda=\lambda_{n_{i}+1}(I)-\lambda_{n_{i}}(I) .
$$


Both $\lambda_{n_{i}}(I)$ and $\lambda_{n_{i}+1}(I)$ may each be obtained in analytical form as a real root of the cubic equation that results from Eq. (5) (with $\operatorname{Re} \epsilon \rightarrow-\tilde{I}_{a}$ and either $n_{0}=n_{i}$ or $n_{0}=n_{i}+$ 1) upon rewriting it in terms of $\lambda(=2 \pi c / \omega)$. Then, substituting the expression for $n_{i}$ in terms of $\lambda_{n_{i}}$, i.e., $n_{i}=$ $\lambda_{n_{i}}\left[\tilde{I}_{a}+u_{p}\left(\lambda_{n_{i}}\right)\right] /(2 \pi \hbar c)$, into the expression for $\lambda_{n_{i}+1}$ on the right-hand side of Eq. (6) yields $\delta \lambda$ as a function of $\lambda_{n_{i}} \equiv \lambda$. Since the exact analytic result for $\delta \lambda$ involves $\tilde{I}_{a}$ and is somewhat cumbersome, we present only its asymptotic expression for the case of $u_{p} \gg \tilde{I}_{a}$ :

$$
\delta \lambda \approx(2 \pi \hbar c) /\left(3 u_{p}\right) \sim \lambda^{-2} .
$$

The approximate result (7) is independent of the binding energy, $\tilde{I}_{a}$; its accuracy depends on both $\lambda$ and $I$ (cf. Fig. 4). Our exact result (6) for $\delta \lambda$ for the $\mathrm{H}$ atom agrees well with the TDSE results of Ref. [6] (where the relation $\delta \lambda \sim \lambda^{-2}$ was obtained using SFA arguments).

The information on the positions of $\lambda_{n_{i}} \equiv \lambda_{i}$ and $\lambda_{n_{i}+1} \equiv \lambda_{i+1}$ enables one to estimate the value of the effective ionization potential, $\tilde{I}_{a}$. By solving the two equations that follow from Eq. (5) for $n_{0}=n_{i}$ and $n_{0}=n_{i}+1$ respectively, one obtains $\tilde{I}_{a}$ in terms of $\lambda_{i}, \lambda_{i+1}$, and the corresponding ponderomotive energies, $u_{p}^{(i)}$ and $u_{p}^{(i+1)}$ :

$$
\tilde{I}_{a}=\left[2 \pi c \hbar+\lambda_{i} u_{p}^{(i)}-\lambda_{i+1} u_{p}^{(i+1)}\right] /\left(\lambda_{i}-\lambda_{i+1}\right) .
$$

Thus, for example, using the TDSE data for the positions of the first two peaks in Fig. 2 of Ref. [6] for hydrogen, we estimate $\lambda_{i}$ and $\lambda_{i+1}$ as $1009 \pm 0.2 \mathrm{~nm}$ and $1031 \pm$ $0.2 \mathrm{~nm}$. The corresponding result for $\tilde{I}_{a}$ lies in the interval $\tilde{I}_{a} \approx 8.4-10.5 \mathrm{eV}$ and is consistent with our choice of $\tilde{I}_{H}$.

To conclude, our study of $P_{\Delta E}(\lambda)$ for an exactly solvable quantum model provides a fully quantum explanation for the results of TDSE-based numerical studies $[3,6]$. Our key finding is that threshold anomalies inherent to multichannel problems strongly affect the smooth powerlike decrease of $P_{\Delta E}(\lambda)$ with increasing $\lambda$ and permit measurement of an effective (reduced) ionization potential in a

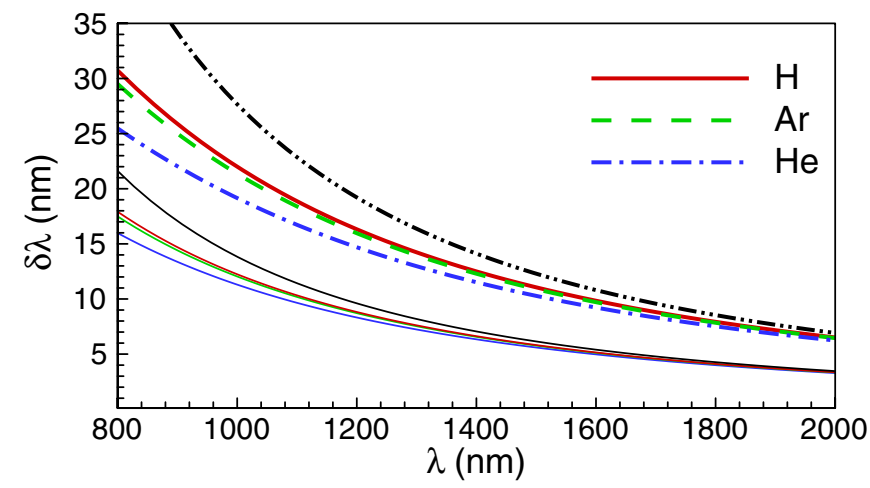

FIG. 4 (color online). Dependence of $\delta \lambda$ on $\lambda$ [cf. Eq. (6)] for intensities $I=1.6 \times 10^{14} \mathrm{~W} / \mathrm{cm}^{2}$ (thick lines) and $I=3.2 \times$ $10^{14} \mathrm{~W} / \mathrm{cm}^{2}$ (thin lines). Dash-dot-dot lines: Eq. (7). laser field. Although TDER results for $P_{\Delta E}(\lambda)$ are exact only for short-range potentials, our fine $\lambda$ scale results give surprisingly good qualitative agreement with the TDSE results [6]. Thus we tentatively conclude that the numerical findings in the benchmark calculations of Ref. [6] provide an important demonstration of intense laser-modified TP in HHG for cases involving a Coulomb potential. In spite of the known fact that SFA results for a zero-range potential unexpectedly reproduce well many key features of ATI and HHG in the plateau region for atoms [14], the similarity of purely quantum, laser-modified TP for both Coulomb and short-range potentials (for which the TP in field-free collision problems are drastically different [8]) is surprising and needs more detailed investigation. We suspect that a strong laser field significantly suppresses the long-range tail of a Coulomb potential, so that not only the plateau features but also the quantum TP in laser-atom processes are governed mostly by the angular momentum of an initially bound electron and by its motion in a strong laser field, rather than by the details of the binding potential.

This work was supported in part by RFBR Grant No. 0702-00574 and by NSF Grant No. PHY-0601196.

[1] T. Brabec and F. Krausz, Rev. Mod. Phys. 72, 545 (2000).

[2] P. Agostini and L. F. DiMauro, Rep. Prog. Phys. 67, 813 (2004); A. Scrinzi, M. Yu. Ivanov, R. Kienberger, and D. M. Villeneuve, J. Phys. B 39, R1 (2006).

[3] J. Tate, T. Auguste, H. G. Muller, P. Salières, P. Agostini, and L. F. DiMauro, Phys. Rev. Lett. 98, 013901 (2007).

[4] M. Lewenstein, Ph. Balcou, M. Yu. Ivanov, A. L'Huillier, and P. B. Corkum, Phys. Rev. A 49, 2117 (1994).

[5] B. Shan and Z. Chang, Phys. Rev. A 65, 011804(R) (2001).

[6] K. Schiessl, K. L. Ishikava, E. Persson, and J. Burgdörfer, Phys. Rev. Lett. 99, 253903 (2007).

[7] A. I. Baz', Zh. Eksp. Teor. Fiz. 33, 923 (1957) [Sov. Phys. JETP 6, 709 (1958)].

[8] L.D. Landau and E. M. Lifshitz, Quantum Mechanics (Pergamon, Oxford, 1980), 4th ed.

[9] M. V. Frolov, A. V. Flegel, N.L. Manakov, and A. F. Starace, Phys. Rev. A 75, 063407 (2007).

[10] M. V. Frolov, N. L. Manakov, E. A. Pronin, and A.F. Starace, Phys. Rev. Lett. 91, 053003 (2003).

[11] M. V. Frolov, A. V. Flegel, N. L. Manakov, and A. F. Starace, Phys. Rev. A 75, 063408 (2007).

[12] M. V. Frolov, A. A. Khuskivadze, N. L. Manakov, and A. F. Starace, J. Phys. B 39, S285 (2006).

[13] W. Becker, S. Long, and J. K. McIver, Phys. Rev. A 50, 1540 (1994).

[14] W. Becker, F. Grabson, R. Kopold, D. B. Milošević, G. G. Paulus, and H. Walther, Adv. At. Mol. Opt. Phys. 48, 35 (2002).

[15] B. Borca, A. F. Starace, A. V. Flegel, M. V. Frolov, and N. L. Manakov, Phys. Rev. A 65, 051402(R) (2002).

[16] N. L. Manakov and M. V. Frolov, Pis'ma Zh. Eksp. Teor. Fiz. 83, 630 (2006) [JETP Lett. 83, 536 (2006)]. 